nicht dem üblichen Standardverfahren entspricht, soweit die notwendige medizinische Abwägung der zu erwartenden Vorteile dieser Methode und ihrer absehbaren und zu vermutenden Nachteile mit denen der standardgemäßen Behandlung die Anwendung rechtfertigt.

4. In diesem Fall ist über die verschiedenen Methoden und ihre jeweiligen Belastungen für den Patien- ten sowie ihre jeweiligen Risiken und Erfolgschancen aufzuklären.

Fehlt es an einer Aufklärung über die angewendete Alternativmethode und verwirklicht sich ein Risiko, das sowohl dieser als auch der Standardmethode, über die aufgeklärt worden ist, immanent ist, so wirkt sich der Mangel der Aufklärung nicht haftungsbegründend aus.

\title{
REZENSIONEN
}

DOI: $10.1007 / \mathrm{s} 00350-011-3014-0$

\section{Menschenwürde und moderne Medizintechnik.}

Herausgegeben von Jan. C. Joerden, Eric Hilgendorf, Natalia Petrillo u. Felix Thiele. (Interdisziplinäre Studien zu Recht und Staat, Bd. 50). Nomos Verlag 2011, 432 S., kart., $€ 89,00$

Der Begriff der Würde des Menschen nach Art. 1 Abs. 1 GG hat „eine enorme praktisch-politische Karriere gemacht“, und so „kann der Diskurs über die Menschenwürde heute von vorneherein nur ein interdisziplinärer Diskurs sein, in dem die Philosophie die verfassungsrechtliche Debatte ebenso rezipiert und verarbeitet, wie umgekehrt die Rechtswissenschaften das philosophische Nachdenken über die Menschenwürde“. Der Philosoph Markus Rothhaar löst diese Prämisse in seinem Beitrag selbst mustergültig ein. Er kommt zu dem Ergebnis, die Menschenwürde bilde einerseits das Prinzip und den Grund aller konkreten Menschenrechte und werde subjektivrechtlich auch durch alle diese Rechte geschützt. Zugleich stelle sie aber auch das Prinzip der Unabwägbarkeit bestimmter unter diesen Rechten dar. Für die bioethische Debatte schließt dieser Autor einen die Menschenwürde zu quantifizieren versuchenden Gradualismus aus. Nicht alle der fünfundzwanzig Beiträge von Verfassern aus Belgien, Deutschland, England, den Niederlanden, der Schweiz und den USA vermögen den juristischen Leser auf gleiche Weise anzusprechen; auch bleibt manche Auskunft problematisch, kontrovers oder gar wenig einleuchtend. Im Ganzen indes bietet der Sammelband eine Reihe von anregenden Ansätzen und Vorschlägen aus der Feder von Medizinern, Biologen, Juristen und Philosophen, die am Bielefelder Zentrum für interdisziplinäre Forschung zusammenwirkten.

Auch die juristischen Stimmen klingen verschieden. So konkretisiert Tatjana Hörnle den Begriff, indem sie die Würde des Menschen verletzt sieht durch ,erhebliche Demütigungen“, von denen nur dann die Rede sein könne, wenn die zu beurteilende Handlung ,einem Subjekt gilt, das in soziale Beziehungen eingebunden ist". Die Bezugnahme auf Menschenwürde sei, so die Autorin durchaus fragwürdig, ,nicht angemessen, wenn es um den Umgang mit Embryonen und Föten geht". Bei der Würde zeige sich, so Edzard Schmidt-Jortzig, „,dass es einen verbindlichen Inhalt dafür kaum gibt“. Es müsse beim Menschenwürdeargument ,unbedingt die Normativitätsquelle offengelegt werden, aus der heraus man argumentiert“. Auch sei ,genau nach den einzelnen Normelementen zu fragen".

Der Sammelband lässt es an resignativen Voten nicht fehlen. Héctor Wittwer meint, ,dass sich medizinethische Kontroversen in der Regel wohl kaum durch die Berufung auf Artikel 1 entscheiden lassen dürften". Jacek Holówka schließt, ,that the concept of dignity is deficient on many counts ... It should not be used ...". Anders etwa Christian Neuhäuser mit dem Ergebnis, ,dass das narrative Verständnis der Menschenwürde durchaus einen Ansatz liefert, um mit den Fragen neuer Medizintechniken umzugehen“. Das Buch weist also eine große Spannweite auf und weckt auch Zweifel: Eine befruchtete Eizelle sei „wahrscheinlich noch kein Mensch“ (S. 231); die „Grenze zwischen Mensch und Tier" sei „willkürlich“ (S. 402). Wer bei Dürig und Böckenförde gelernt hat, wird das Buch mit Sorgenfalten aus der Hand legen. Gewiss verlangen die enormen naturwissenschaftlichtechnischen Fortschritte der Postmoderne neue juristische und philosophische Bescheide. Aber wenn es unverrückbare äußerste rechtliche und ethische Grenzen des Machbaren geben soll, müssen sie

Prof. Dr. iur. Dr. h.c. Adolf Laufs, Heidelberg, Deutschland herausgearbeitet werden; dafür bleibt auch nach dem Erscheinen des Sammelbandes noch einiges zu tun.

\section{Honorararzt - Flexibilität und Freiberuflichkeit.}

Herausgegeben von Nicolai Schäfert. Verlag Springer, Berlin 2010, XXV u. 164 S., kart, $€ 39,95$

Die Verzahnung ambulanter und stationärer Betreuung von Patienten hat der Gesetzgeber durch Anpassung der Kooperationsbedingungen (Wegfall der Unvereinbarkeitsvorschrift des $₫ 21$ Abs. 2 Ärzte-ZV) und Organisationsformen erheblich erleichtert. Die meisten dieser Neuerungen sind in der Fachliteratur ausreichend dargestellt und von der Rechtsprechung teilweise konkretisiert; auch Chancen und Risiken, speziell der Zusammenarbeit zwischen Vertragsärzten und Krankenhäusern, sind erläutert (s. insbes. Makoski, GesR 2009, 225; Quaas, GesR 2009, 462; Dahm, MedR 2010, 597). Weitgehend unberücksichtigt blieb dabei die Figur des sog. ,Honorararztes“. Diesem Thema widmet sich ein Team von 26 (!) Autoren unterschiedlicher Fachrichtungen in der hier besprochenen Veröffentlichung. Die einzelnen Beiträge sind kurz. Sie beleuchten überwiegend die Chancen der selbständigen Tätigkeit von Ärzten (ohne oder mit anderweitigen arbeitsrechtlichen Verpflichtungen; also Privatärzte, Vertragsärzte, angestellte Ärzte, aber auch Ärzte ohne berufliche Bindung) für Krankenhäuser, MVZs, Forschungseinrichtungen, aber auch öffentliche Einrichtungen des Gesundheitswesens, des Rettungsdienstes etc. Gegenwärtig greifen insbes. Anästhesisten auf diese Form zurück. Dargestellt werden u. a. Sicht und Erwartungen der Auftraggeber (S. 4ff.) sowie einige Vor- und Nachteile honorarärztlicher Tätigkeit (S. 16f.). RA Willems (S. 20 ff.) beschreibt den juristischen Status des Honorararztes und seiner Arbeit als selbständige Tätigkeit; er erläutert die Verträge dazu und zeigt Folgerungen für das Berufsrecht sowie das Haftungsrecht auf und erörtert Fragen der Versicherung. Ein besonderes Kapitel (S. 93 ff.) ist der "Qualität im Honorararztwesen" gewidmet (Autor: der Herausgeber Schäfer). Im Anhang finden sich Schilderungen über das Honorararztwesen im Ausland. Kopiervorlagen und Musterbögen sind ebenfalls neben einem (knappen) Literaturverzeichnis angehängt.

Das Buch betrifft Kernbereiche des Honorararztes in der Praxis; die z. T. komplizierten vertragsarztrechtlichen und berufsrechtlichen Fragen und ihre Probleme in der praktischen Umsetzung (z.B.: evt. Vorteilsgewährungen) werden nur gestreift. Der Leser ist daher darauf angewiesen, dazu ergänzend aktuelle Handbücher mit Literatur und Rechtsprechung hinzuzuziehen, um bei seiner Verdingung als Honorararzt Fehler zu vermeiden. Die Autoren sehen in der Figur des Honorararztes überwiegend erhebliche Chancen. Nur an wenigen Stellen klingt an, dass die oft nur kurzfristige Beschäftigung von Honorarärzten einen Verlust an Qualität und Patientenbindung nach sich ziehen und auch Unruhe unter den „Stammmitarbeitern“ bewirken könnte. Auf Dauer lassen sich mit Honorarärzten die Strukturprobleme - vorwiegend der großen Krankenhäuser - kaum nachhaltig lösen (s. S. 15). Indessen kommt der Honorararzt einer zunehmenden workand-life-Philosophie der nachwachsenden Generation (vgl. z. B. Friebe/Lobo [Hrsg.], Wir nennen es Arbeit. Intelligentes Leben jenseits der Festanstellung, 2. Aufl. 2008) in der Medizin entgegen.

Rechtsanwalt Dr. iur. Gernot Steinhilper, Wennigsen, Deutschland 